\title{
Guide de bonnes pratiques Cliniques et biologiques en Assistance Médicale à la Procréation
}

\section{Commission Nationale de Médecine et de Biologie de la Reproduction et du Diagnostic Prénatal}

Ministère de l'emploi et de la solidarité - Arrêté du 12 Janvier 1999, publié au J.O. du 28 février 1999, pages 3061 à 3069 .

\section{1 - DISPOSITIONS COMMUNES À L'ENSEMBLE DES PRATIQUES D'AMP}

\section{1. - Fonctionnement et organisation des centres}

\subsection{1- Organisation}

Les actes cliniques ou biologiques d'AMP sont effectués sous la responsabilité d'un ou plusieurs praticiens nommément agréés pour une ou plusieurs des activités cliniques ou biologiques.

Dans le cas où plusieurs praticiens sont agréés pour les activités cliniques, ils désignent parmi eux un coordinateur. Il en est de même pour les activités biologiques. Dans le cas où un seul praticien est agréé, il assure cette tâche.

Le coordinateur :

- veille au respect des dispositions législatives et réglementaires qui s'attachent à ces activités,

- établit le rapport d'activité prévu à l'article $\mathrm{L}$. 184-2 du CSP,

- veille à l'animation de l'équipe,

- veille également à ce que le personnel qui participe à la mise en oeuvre de l'AMP dispose des compétences spécifiques nécessaires. Ces compétences doivent être régulièrement actualisées par des formations appropriées.

L'ensemble des cliniciens et biologistes participant à l'activité d'AMP constituent une équipe pluridisciplinaire, tel qu'il est mentionné à l'article L. 152-10 du CSP, équipe à laquelle doivent s'adjoindre, en tant que de besoin, un psychiatre ou un psychologue, un généticien et un andrologue. Elle a pour rôle, outre les missions mentionnées à l'article L. 152-10 (vérification de la motivation et information des couples), de discuter préalablement à toute $\mathrm{AMP}$, au cours de réunions régulières : son indication, les protocoles de stimulation, le choix de la technique d'AMP.

Doivent aussi être discutés, si nécessaire, la poursuite, la modification ou l'arrêt du protocole d'AMP en cours.

De manière plus générale, le rôle de l'équipe pluridisciplinaire comprend également : l'organisation générale des activités, les orientations thérapeutiques et la discussion des problèmes éthiques.

Un compte rendu de chaque réunion sera établi et conservé au moins 3 ans. Les décisions individuelles seront consignées dans le dossier médical des patients.

Aucun patient ne peut être pris en charge en vue d'AMP dans les établissements autorisés 
sans cette évaluation préalable par l'équipe pluridisciplinaire. Ces dispositions s'appliquent à l'ensemble des actes d'AMP, y compris l'IAC.

Dans le cadre de l'IAC, la concertation implique, au minimum, le biologiste agréé et le correspondant clinique.

\subsection{2. - Conditions techniques de fonction- nement}

\section{- LOCAUX}

a) Toute structure clinique autorisée à pratiquer les activités cliniques d'AMP doit comprendre au minimum (R. 184-1-5 du CSP) :

- une pièce pour les entretiens avec les couples,

- un bloc opératoire,

- une salle de ponction située à proximité du bloc opératoire et équipée d'un matériel permettant la pratique d'une anesthésie générale et d'une réanimation en cas de besoin,

- une pièce destinée au transfert des embryons, - une salle de réveil conforme au décret $n^{\circ} 94-$ 1050 relatif aux conditions techniques de fonctionnement des établissements de santé en ce qui concerne la pratique de l'anesthésie,

- des lits d'hospitalisation.

b) Tout laboratoire autorisé à pratiquer les activités biologiques d'AMP doit comprendre au minimum, en fonction des activités pour lesquelles il est autorisé (R. 184-1-9 du CSP).

* Pour l'ensemble des activités biologiques $d^{\prime} A M P$

- Une pièce exclusivement affectée au recueil de sperme. Cette pièce doit être équipée d'un lavabo équipé d'un dispositif automatique permettant l'arrivée d'eau sans intervention manuelle, et de dispositifs permettant d'assurer l'hygiène du recueil, avec affichage des procédures. La pièce doit offrir toutes les garanties de confidentialité.

- Une pièce exclusivement affectée au traitement des gamètes en vue d'AMP ; cette pièce ne peut en aucun cas être utilisée pour des analyses de biologie médicale, y compris pour la spermiologie. Lorsque sont effectués des activités de FIV la pièce doit être équipée d'un sas.
* Pour les activités de conservation de gamètes et d'embryons

- Une pièce exclusivement affectée à cette activité ; cette pièce doit être équipée d'un dispositif d'extraction de vapeur d'azote. Elle doit être également équipée d'une protection contre le vol.

En outre, il est recommandé de disposer d'une pièce pour les entretiens avec les couples et d'un bureau de secrétariat et d'archives.

Les locaux doivent être de taille suffisante au regard de l'activité.

\section{- MATÉRIEL}

a) Toute structure de gynécologie ou gynécologie-obstétrique autorisée à pratiquer le recueil par ponction d'ovocytes doit être équipée dans ses locaux, au minimum, du matériel suivant.

- Un échographe de haute définition avec sonde vaginale (R. 184-1-5 du CSP)

- Un matériel de ponction et recueil ovocytaire à usage unique, la sonde étant protégée de manière efficace sur toute sa longueur afin d'assurer la sécurité sanitaire

- Un système permettant le maintien des prélèvements à $37^{\circ} \mathrm{C}$ jusqu'à leur arrivée au laboratoire.

\section{b) Toute structure autorisée à pratiquer le recueil par ponction de spermatozoüdes} doit être équipée, au minimum, du matériel suivant.

- Un matériel de ponction à usage unique

- Un système permettant le maintien des prélèvements entre $20^{\circ} \mathrm{C}$ et $35^{\circ} \mathrm{C}$ jusqu'à leur arrivée au laboratoire.

c) Tout laboratoire autorisé à pratiquer les activités biologiques d'AMP doit être équipé au minimum du matériel suivant.

* Pour le traitement du sperme en vue d'insémination:

- Un matériel permettant des conditions d'asepsie rigoureuse (hotte à flux laminaire ou matériel équivalent) 
- Une centrifugeuse

- Un microscope

- Une étuve ou un bain-marie à $37^{\circ} \mathrm{C}$.

* Pour la fécondation in vitro :

Outre le matériel nécessaire pour le traitement du sperme en vue d'insémination

- Un microscope inversé avec platine chauffante ou enceinte thermostatée à $37^{\circ} \mathrm{C}$,

- Un stéréomicroscope équipé de platine et plateau chauffant, thermostatés à $37^{\circ} \mathrm{C}$,

- Une étuve à $\mathrm{CO} 2$

- Pour la FIV avec micromanipulation, un dispositif de micromanipulation adapté sur le microscope inversé.

* Pour la congélation et la conservation du sperme

- Une cuve de stockage des paillettes de sperme dans l'azote liquide équipée de visotubes et canisters ou équivalent,

- Une réserve d'azote liquide avec dispositif de remplissage des cuves.

En outre, il est souhaitable, en cas d'activité importante, que le laboratoire dispose d'appareils permettant le conditionnement automatique du sperme en paillettes et la congélation programmée du sperme dans les vapeurs d'azote.

* Pour la congélation et la conservation des embryons

- Un appareil permettant la congélation programmée des embryons dans les vapeurs d'azote

Une cuve de stockage des paillettes contenant les embryons ; cette cuve doit être exclusivement affectée à cet usage.

Une cuve d'azote de dépannage afin d'assurer la sécurité en cas d'incident technique.

\section{- RÈGLES D'HYGIÈNE ET DE SÉCURITÉ AU LABORATOIRE}

a) L'ensemble du petit matériel en contact avec les gamètes et les embryons doit être à usage unique. Lorsqu'il est fourni non stérile, il doit être stérilisé. Les modalités de la stérilisation doivent faire l'objet d'une procédure écrite et validée.

b) Les milieux de culture et réactifs dont l'utilisation est décrite au chapitre 2.2.2., préparés ou reconstitués au laboratoire doivent porter la date de leur préparation et celle de leur péremption. Les réactifs d'origine industrielle doivent comporter la date de leur réception au laboratoire et, le cas échéant, la date limite d'utilisation. Ils doivent être conservés dans des conditions appropriées, respectant les normes indiquées par les fabricants. La date de péremption des milieux et réactifs doit être vérifiée une fois par mois. La date de la dernière vérification doit être notée dans le cahier de préparation. Les réactifs périmés seront éliminés.

c) Les appareils utilisés doivent être régulièrement et efficacement nettoyés, décontaminés, entretenus et contrôlés. L'ensemble de ces opérations ainsi que les visites d'entretien réalisées par le constructeur ou par un organisme de maintenance, doivent être notés dans un document affecté à chaque appareil. Des procédures de secours doivent être prévues en cas de panne ou de dysfonctionnement, notamment l'accès à la cuve de dépannage, préalablement identifiée, pour la conservation des gamètes et des embryons. Le matériel doit être renouvelé régulièrement en fonction de son obsolescence et de son usure.

d) Dans le cadre des précautions " universelles" à respecter pour les laboratoires travaillant sur des prélèvements humains, définies par le CDC d'Atlanta (MMWR n ${ }^{\circ} 2$ spécial, 1987), le personnel du laboratoire doit suivre les règles suivantes:

- port de gants pour toutes les manipulations de produits humains, à changer chaque fois que nécessaire et nécessairement entre 2 patients,

- port de masque pendant les manipulations.

- lavage des mains et de la peau immédiat en cas de projection,

- prévention des piqûres et coupures (collecteurs, interdiction de reboucher les aiguilles)

Il est rappelé que la vaccination du personnel du laboratoire contre l'hépatite B est obligatoire. 
e) Les locaux doivent faire l'objet d'un nettoyage et d'une désinfection efficaces quotidiennes afin d'éviter tout risque de contamination. Les procédures d'entretien doivent être décrites et notées sur un cahier spécifique. Les produits utilisés à cet effet doivent être non toxiques.

\subsection{3. - DOCUMENTS}

\section{- DOSSIER MÉDICAL}

L'équipe clinique et l'équipe biologique doivent être chacune en possession des pièces exigées avant le recours à l'AMP. En outre, l'équipe clinique doit disposer d'un résumé du dossier biologique et l'équipe biologique d'un résumé du dossier clinique. Ces dossiers doivent être conservés pendant une durée minimale de 30 ans.

Ce dossier comprend :

- Les pièces exigées avant le recours à l'AMP,

- Les éléments cliniques et biologiques communs à toute AMP,

- Les éléments cliniques et biologiques spécifiques de chaque AMP,

- Le double d'un compte-rendu de la tentative remis aux patients.

Les règles concernant le contenu et la conservation du dossier du donneur ou de la donneuse sont traitées en 3.2.3.

- Les pièces exigées avant le recours à l'AMP (L. 152-2 du CSP) sont :

- Un justificatif du mariage ou d'une durée de vie commune d'au moins 2 ans,

- Le consentement écrit du couple préalable à la mise en oeuvre de l'AMP, et renouvelé avant chaque transfert d'embryons ou chaque insémination.

\section{- Les éléments cliniques et biologiques} communs à toute $A M P$ sont :

- L'indication de l'AMP, accompagnée des éléments qui ont permis de l'établir,

- Les résultats des tests relatifs à la sécurité sanitaire,

- Le détail de l'ensemble des traitements administrés et la réponse ovarienne à la stimulation,
- Les techniques détaillées d'AMP utilisées à chaque étape,

- En cas de grossesse, une information sur l'évolution de celle-ci et sur l'état de santé du ou des nouveau-nés.

\section{- Les éléments cliniques et biologiques de l'IAC sont:}

- La détermination du moment estimé de l'ovulation,

- Les paramètres du sperme utilisé pour l'insémination et notamment, le nombre de spermatozoïdes mobiles inséminés,

- La date et l'heure du recueil de sperme, de sa préparation et de l'insémination.

\section{- Les éléments cliniques et biologiques de la FIV sont :}

- La date de la ponction (et son compte-rendu) et le nombre d'ovocytes recueillis et inséminés,

- La qualité ovocytaire (éventuellement atrésie, zone pellucide endommagée),

- Les techniques spécifiques éventuellement mises en oeuvre (micromanipulation, coculture, éclosion assistée, etc.),

- Le nombre de spermatozoïdes utilisés pour l'insémination in vitro,

- Le nombre d'ovocytes fécondés le lendemain de l'insémination, incluant les éventuelles fécondations anormales,

- Le nombre et la morphologie des embryons obtenus et leur stade de développement, le surlendemain de l'insémination et au moment du transfert, si celui-ci est différé,

- La date et le nombre d'embryons transférés,

- Le nombre d'embryons congelés,

- Le compte rendu de la tentative doit comprendre au minimum le nombre d'ovocytes recueillis, inséminés, le nombre d'embryons total obtenus, transférés et congelés.

\section{- REGISTRES ET RELEVÉS CHRONOLO. GIQUES}

Ces documents doivent être conservés dans le respect de la confidentialité. Ils doivent être reliés et numérotés. Ils doivent être gardés à proximité des locaux où sont réalisées les 
diverses activités qu'ils concernent (laboratoire de FIV, lieu de conservation des gamètes et embryons).

\section{a) Relevé des activités cliniques}

Les établissements de santé doivent noter sur un registre toutes les prises en charge pour FIV, dès le début des cycles de stimulation, les causes d'arrêt éventuelles, la date de la ponction, le résultat de la tentative et l'issue de la grossesse.

\section{b) Relevé chronologique des tentatives d'AMP}

Etabli par les biologistes, il doit comporter au minimum la date de l'AMP (date de l'insémination pour les IAC, date de la ponction d'ovocytes pour les FIV), l'identité du couple, le nom du centre clinique, la qualité des intervenants, la technique d'AMP mise en œuvre et les milieux produits et matériels utilisés ainsi que le résultat de la tentative, ceci afin d'obtenir la traçabilité des procédures mises en œuvre pour chaque tentative.

\section{c) Registre de conservation des gamètes}

Son contenu est précisé à l'article R. 184-2-1 du CSP. Chaque registre doit mentionner l'identité de la personne à l'origine des gamètes (nom $\mathrm{du}$ patient en cas d'autoconservation, code d'identification du donneur en cas de don), les lieux et dates de congélation des gamètes. Il comporte aussi les indications précises du lieu de conservation des gamètes dans la cuve affectée à cet effet. Enfin, il doit préciser les dates et les modes d'utilisation des gamètes, et, le cas échéant, l'identité du couple destinataire du don de gamètes.

\section{d) Registre de conservation des embryons}

Son contenu est fixé par l'article R. 184-2-2 du CSP. Chaque registre doit mentionner l'identité du couple à l'origine de l'embryon (et le cas échéant le code d'identification du donneur de gamètes), le lieu et les dates de fécondation et de congélation et, le cas échéant, le lieu de conservation antérieur.

Pour chaque couple il convient de noter le nombre d'embryons conservés, les indications précises du lieu de conservation des embryons dans la cuve affectée à cet effet, et les informations relatives au devenir de tous les embryons (date de décongélation, date de transfert, aspect morphologique à la décongélation, date d'arrêt éventuel de la conservation) ainsi que le résultat de tous les transferts.

Enfin, il est nécessaire d'y faire figurer les dates des relances effectuées auprès des couples sur le point de savoir s'ils maintiennent leur demande parentale, et le résultat de celles-ci.

\section{e) Cahier de procédures}

Un cahier de procédures est mis en place dans chaque unité clinique et laboratoire. Les procédures, tenues à jour, sont dactylographiées, datées et validées par le ou les responsables agréés, et signées par l'ensemble des intervenants.

\subsection{4. - CONFIDENTIALITÉ ET SÉCURITÉ}

\section{- LOCAUX, DOCUMENTS.}

La pièce affectée à la conservation des gamètes et des embryons, ainsi que celle contenant les registres doit être équipée d'une protection contre le vol. L'accès à ces locaux est placé sous la responsabilité des praticiens agréés. Les procédures de sécurité concernant la pièce affectée à la conservation des gamètes et des embryons sont écrites.

Les documents réglementaires sont gardés dans des conditions de sécurité garantissant une conservation de longue durée.

La communication d'éléments concernant les dossiers est placée sous la responsabilité des cliniciens et biologistes agréés.

\section{- INFORMATIQUE}

Les ordinateurs contenant les données nominatives ne doivent pas être reliés à un système extérieur à l'établissement, sauf accord de la Commission Nationale Informatique et Liberté (CNIL). L'accès aux informations nominatives est placé sous la responsabilité des responsables agréés et réservé aux personnes autorisées par le biais de codes d'accès confidentiels.

Des procédures sont écrites pour notifier les modalités d'introduction ou de modification des données nominatives ainsi que leur consultation. Il est indispensable de disposer d'une 
double conservation des données.

\section{2. - ETAPES PRÉALABLES À LA MISE EN OEUVRE DE L'AMP}

La décision d'AMP ne devra être prise qu'après avoir effectué un bilan médical complet du couple et après l'avoir informé, le cas échéant, d'autres possibilités thérapeutiques ainsi que de la faculté de recourir à l'adoption.

1.2.1. - CADRE LÉGAL DE LAMP (ART. L. 152-2 CSP)

L'AMP doit toujours répondre à une demande parentale, pour remédier à une infertilité dont le caractère pathologique a été médicalement constaté ou pour éviter la transmission à l'en. fant d'une maladie d'une particulière gravité. Cette dernière indication correspond principalement à l'AMP avec don de gamètes, à l'accueil d'embryon ou à la FIV avec diagnostic pré-implantatoire si la maladie est considérée de plus comme incurable. Aucune AMP ne peut être réalisée pour des raisons de convenance personnelle.

La prise en charge ne peut concerner qu'un couple formé d'un homme et d'une femme, en âge de procréer.

Le couple doit être marié ou en mesure d'apporter la preuve d'une vie commune depuis au moins deux ans. Il appartient à l'équipe médicale de demander la production des documents attestant de l'identité des deux membres du couple, de la réalité du mariage (livret de famille, fiche récente d'état civil) ou de la durée de vie commune (par tout moyen).

Les deux membres du couple doivent être vivants au moment de la réalisation de l'AMP (insémination ou transfert d'embryon). En pratique, le respect de cette condition est assuré par l'exigence d'un double consentement signé au moment de chacune de ces opérations.

\subsection{2. - INDICATIONS ET DIAGNOSTIC}

Lorsqu'elle a pour but de remédier à l'infertilité, l'AMP est réservée à des couples pour lesquels les chances de fécondation naturelle sont nulles ou très faibles. Certaines indications sont absolues, comme l'absence ou l'occlusion bilatérale des trompes (FIV conventionnelle) ou les stérilités masculines par azoospermie
(ICSI, IAD).

En revanche, certaines indications sont relatives, notamment toutes celles qui correspondent à une simple hypofertilité, et doivent être jugées en fonction du bilan diagnostique, de l'âge des patients et de la durée d'infécondité. Il en est ainsi des altérations tubaires, des anomalies du spermogramme, des anticorps antispermatozoïdes, de l'endométriose, des anomalies de la glaire cervicale et des infécondités sans cause diagnostiquée (idiopathiques).

En cas d'indication relative, il est recommandé d'observer un délai raisonnable avant la mise en cuvre de l'AMP, délai dont l'importance dépend de l'âge des patients et du temps écoulé depuis qu'ils essaient d'obtenir une grossesse. En effet, dans ce cas, la plus grande partie des couples concevront spontanément. Il n'est de ce fait pas raisonnable de proposer une FIV à un couple dont la femme a moins de 35 ans sans une durée d'infécondité d'au moins deux ans. Ce délai peut être raccourci ensuite. Cependant, compte tenu de la faible efficacité des techniques au delà de 37 ans, il est recommandé de ne prendre en charge des femmes plus âgées que dans les cas où la fonction ovarienne a été jugée satisfaisante.

En ce qui concerne l'exploration de l'infécondité, il est inutile d'observer un délai d'attente avant de la mettre en œuvre si l'anamnèse fait envisager l'existence de troubles de la fertilité.

\section{Explorations préalables}

La réalisation d'une AMP chez un couple infertile ne peut être envisagée sans un bilan comprenant au moins les examens suivants :

- Examen clinique complet des 2 partenaires,

- Exploration du cycle comprenant au minimum une courbe de température et un dosage de FSH et oestradiol en début de cycle (J2 à J5),

- Hystérosalpingographie

- Spermogramme, spermocytogramme, spermoculture,

Ce bilan minimum pourra être complété en fonction du contexte clinique, afin de mieux cerner le diagnostic, le pronostic de fertilité, l'attitude thérapeutique à adopter. 
La constatation d'une anomalie du spermogramme selon les critères de l'OMS (concentration inférieure à 20 millions $/ \mathrm{ml}$, mobilité totale inférieur à $40 \%$ ou mobilité progressive inférieure à $25 \%$, taux de formes normales inférieur à $30 \%$ ) doit orienter vers une exploration andrologique.

En cas d'azoospermie, ou d'oligozoospermie sévère faisant envisager une ICSI, il est nécessaire de disposer d'un bilan spermatique approfondi comportant au moins un test de migration - survie indiquant le nombre total de spermatozoïdes mobiles et vivants au recueil et après $24 \mathrm{~h}$ d'incubation. D'autres explorations sont à prévoir en fonction du contexte clinique. Une enquête génétique est indispensable dans la plupart des cas

A l'issue de ce bilan, une stratégie thérapeutique pourra être proposée : technique retenue, nombre total de tentatives, alternatives en cas d'échec.

\subsection{3. - ENTRETIENS DU COUPLE} DEMANDEUR AVEC L'ÉQUIPE MÉDICALE PLURIDISCIPLINAIRE. (ART L 15210 CSP)

Préalablement à la mise en œuvre de l'AMP, le couple demandeur doit rencontrer un clinicien et un biologiste de l'équipe pluridisciplinaire. Il doit pouvoir également, sur sa demande ou celle des praticiens, rencontrer tout autre membre de l'équipe, y compris le médecin qualifié en psychiatrie ou le psychologue dont l'établissement s'assure le concours. Il peut être fait appel en cas de besoin à un service social.

\section{a) Information du couple}

Une information claire, précise et adaptée doit être donnée au couple au cours de ces entretiens sur la cause de son infécondité ainsi que sur le choix de la ou des techniques envisagées. Chaque technique doit être présentée avec ses chances de succès pour le couple, sa pénibilité ainsi que les contraintes, notamment d'ordre matériel, financier ou psychologique, qu'elle peut entraîner. Doivent être exposés les risques potentiels à court et à long terme, liés notamment à la stimulation ovarienne, au geste chirurgical, aux techniques biologiques utilisées ainsi que le risque de grossesses mul- tiples. Une information sur les éventuelles solutions alternatives devra être délivrée : chances de concevoir spontanément, chirurgie des trompes ou des voies excrétrices masculines. De même, l'éventualité de devoir arrêter la prise en charge devra être envisagée d'emblée.

Une information doit aussi être délivrée sur les possibilités ouvertes par l'adoption (conditions légales, modalités pratiques, adresse des organismes).

Le couple doit être informé de l'état actuel et encore limité des connaissances concernant l'état de santé des enfants nés après AMP. Le praticien doit encourager le couple à participer aux études épidémiologiques qui pourront lui être proposées.

La remise d'un dossier guide explicatif devra compléter ces informations.

\section{b) Vérification de la motivation du couple}

Le praticien s'assure de la réalité du désir d'enfant chez la femme et chez l'homme. Il vérifie l'absence d'obstacle grave à la mise en œuvre de l'AMP dans l'intérêt de l'enfant à naître. Si nécessaire, après entretien avec le psychiatre ou le psychologue et concertation au sein de l'équipe, la mise en œuvre de l'AMP pourra être différée.

\section{c) Confirmation de la demande}

L'AMP ne peut être mise en œuvre sans que les 2 membres du couple aient confirmé par écrit leur demande. Cette confirmation ne peut intervenir qu'à l'issue d'un délai d'un mois à compter du dernier entretien.

\subsection{4. - Tests de sécurité sanitaire}

\section{a) AMP intra-conjugale}

Une bonne pratique médicale implique la réalisation de certains tests sanitaires avant la mise en œuvre d'une AMP intra-conjugale pour éviter le risque de contamination de prélèvements d'autres couples, du personnel et de l'enfant à naître. Il est nécessaire de rechercher des marqueurs biologiques d'infection et, lorsque cela est techniquement possible, d'infectivité par VIH1, VIH2, les virus des hépatites $\mathrm{B}$ et $\mathrm{C}$ et la syphilis chez les 2 membres du couple. Cette recherche doit être réalisée 
avant la première tentative et, pour les autres tentatives, si le délai depuis la dernière détermination est supérieur à 12 mois.

En ce qui concerne l'infection par le VIH, la prise en charge des couples ne peut se faire que dans le cadre d'un protocole de recherche clinique pluridisciplinaire, relevant des prescriptions de la loi Huriet comprenant l'avis d'un CCPPRB et validé par la CNMBRDP.

En ce qui concerne le virus de l'hépatite $B$, si l'homme est porteur de l'antigène HBS, il conviendra de vacciner la conjointe séronégative préalablement à toute AMP. Si la femme est porteuse de l'antigène HBS, l'AMP est possible, mais le couple doit être averti de la nécessité de réaliser la sérovaccination spécifique de l'enfant à la naissance.

En cas de séropositivité avec virémie de l'un ou l'autre ou des 2 membres du couple vis-à-vis de l'hépatite $\mathrm{C}$, la prise en charge des couples est uniquement possible dans le cadre d'un protocole de recherche clinique . pluridisciplinaire, relevant des prescriptions de la loi Huriet comprenant l'avis d'un CCPPRB et validé par la CNMBRDP

En cas de séropositivité pour la syphilis, l'AMP doit être précédée d'un traitement spécifique.

\section{b) AMP avec don}

Des règles de sécurité sanitaire sont obligatoires (articles R. 673-5-10 à R. 673-5-13 du CSP) pour le recueil et l'utilisation de gamètes humains provenant de dons en vue de la mise en cuvre d'une assistance médicale à la procréation. Un certain nombre d'analyses de biologie médicale doivent être pratiquées chez le donneur de gamètes avant le don, à la recherche des marqueurs biologiques d'infection et, lorsque cela est techniquement possible, d'infectivité :

(1) pour les virus VIH1, VIH2, HTLV1, HTLV2, hépatites $\mathrm{B}$ et $\mathrm{C}$, et pour la syphilis.

(2) pour le cytomégalovirus (dosages d'IgM et $\operatorname{IgG).}$

- Si les résultats sont positifs à l'une des infections citées au (1), les donneurs ou les donneuses de gamètes ne peuvent être retenus. S'ils sont négatifs, un délai de 6 mois doit être respecté pendant lequel le sperme provenant du don ne peut pas être cédé, et les embryons issus d'ovocytes cédés ne peuvent pas être transférés. A l'issue de ce délai, les textes réglementaires prévoient que le bilan soit renouvelé pour les infections (1), à l'exception de HTLV1 et HTLV2. Il est cependant recommandé de les pratiquer. Le praticien est tenu de s'assurer que les résultats des analyses sont demeurés négatifs. Dans le cas où l'un ou plusieurs de ces résultats seraient devenus positifs, les gamètes ne pourraient être cédés ni les embryons transférés.

En ce qui concerne le CMV (2), si le premier test est positif, il est préférable, en l'absence d'IgM, et après la période réglementaire de 6 mois, de n'utiliser les gamètes que pour les couples receveurs dont l'un au moins des membres est positif pour le CMV. En présence d'IgM, le don est récusé. Si le premier test est négatif, il doit être répété à l'issue de la période de 6 mois. Lorsque le deuxième test de CMV se positive, traduisant une infection récente, le don est récusé.

Enfin, il est préférable de réserver les gamètes provenant de donneurs négatifs pour le CMV à des couples receveurs dont les 2 membres sont eux-mêmes négatifs.

Outre les analyses ci-dessus il est nécessaire, en cas de don de sperme, de pratiquer une spermoculture à chaque recueil de sperme.

Enfin, ne peuvent être retenus les donneurs de gamètes dont l'interrogatoire révèle un risque potentiel de transmission de la maladie de CREUTZFELDT-JAKOB ou d'autres encéphalopathies subaiguës spongiformes (antécédents familiaux de cette maladie, administration de produits extractifs humains provenant du cerveau - notamment hormone de croissance -, explorations neurochirurgicales invasives). L'interrogatoire doit comprendre des questions concernant ces problèmes, et il est conseillé de mentionner les réponses dans le dossier des patients.

Un document, nécessaire pour la cession des gamètes et des embryons issus des ovocytes cédés, doit être établi par le biologiste agréé précisant : 
- le nom et l'adresse de l'établissement autorisé ayant recueilli ces gamètes et le cas échéant, le nom et l'adresse de l'établissement autorisé ayant procédé à la fécondation in vitro des ovocytes cédés.

- les résultats des analyses ci-dessus sans aucune mention permettant d'identifier les donneurs de gamètes.

- l'identité du couple destinataire des gamètes.

Le praticien mettant en œuvre l'AMP avec tiers-donneur est tenu au préalable de se faire remettre le document cité ci-dessus et de prendre connaissance des résultats des analyses. Il paraît souhaitable qu'il appose sa signature sur ce document.

\section{2 - DESCRIPTION DES PRATIQUES D'AMP INTRA-CONJUGALE}

\section{1. - INSÉMINATION ARTIFICIELLE AVEC SPERME DE CONJOINT}

Les IAC constituent une AMP à part entière, dont les indications doivent être soigneusement pesées, et concernent essentiellement les infécondités d'origine cervicale, inexpliquée ou masculine. Dans tous les cas, l'indication ne sera posée qu'après réalisation d'un test de séparation des spermatozoïdes. Ce test doit montrer que le nombre total de spermatozoïdes mobiles disponibles est supérieur à 500000 , après préparation de la totalité de l'éjaculat. Il est recommandé que le test de séparation soit pratiqué dans le laboratoire préparant les spermatozoïdes en vue d'insémination.

Dans certains cas particuliers, l'insémination sera réalisée avec des paillettes de sperme autoconservé.

La mise en œuvre de cette technique nécessite, comme pour toute AMP, un entretien préalable du couple demandeur avec le clinicien amené à la réaliser. Au cours de cet entretien le clinicien s'assure du respect des conditions légales (article L. 152-2 du CSP), donne l'information légale au couple (article L. 152-10 du CSP) et recueille le consentement signé. Celui ci devra être confirmé à chaque insémination.

L'insémination est le plus souvent réalisée par voie intra-utérine après préparation du sper- me dans un laboratoire autorisé.

\subsection{1. - COORDINATION CLINICIEN - BIOLOGISTE}

La coordination entre le clinicien et le biologiste est indispensable. Le biologiste doit donner son accord avant la mise en œuvre de l'IAC. Il ne peut prendre en charge la préparation du sperme que s'il a une information complète sur:

- L'indication de l'IAC,

- Les données détaillées d'un spermogramme datant de moins de 3 mois et d'un test de séparation des spermatozoïdes mobiles (acte réservé aux laboratoires agréés par l'AMP),

- Le respect des conditions légales (Cf. 1.2.1.),

- La signature du consentement à l'IAC qu'il doit conserver,

- Les résultats des tests de sécurité sanitaire (sérologies VIH, hépatites B et C, et syphilis).

Il doit impérativement s'assurer auprès du clinicien des résultats de chaque cycle en cours ainsi que de l'issue d'une grossesse éventuelle, et recueille le bilan d'activité des cliniciens avec lesquels il collabore.

\subsubsection{ASPECTS CLINIQUES}

L'insémination est généralement précédée d'une stimulation de la croissance folliculaire, en vue d'obtenir un petit nombre de follicules matures (1 à 3 au maximum). Un monitorage de l'ovulation échographique et hormonal (oestradiol, LH) est donc nécessaire permettant de surseoir à l'insémination si plus de 3 follicules mûrs sont mis en évidence. Sauf exception, une insémination par cycle est suffisante. Le nombre de cycles d'inséminations ne devrait pas dépasser six.

\subsubsection{ASPECTS BIOLOGIQUES}

Le recueil de sperme en vue d'AMP doit avoir lieu dans un laboratoire d'AMP autorisé.

Le sperme doit être recueilli par masturbation dans des conditions d'asepsie rigoureuse, après lavage et désinfection des mains et du gland. Dans certains cas (anticorps anti-spermatozoïdes, nécrozoospermie) le recueil direct dans un milieu de culture est nécessaire. Les procé- 
dures d'hygiène du recueil doivent être affichées dans la pièce destinée au recueil.

Pour des raisons de sécurité sanitaire, il est de bonne pratique de traiter le sperme sous une hotte à flux laminaire avec du matériel à usage unique, des milieux stériles et contrôlés (de préférence à $37^{\circ} \mathrm{C}$ ) et répondant aux exigences réglementaires en vigueur (voir chapitre 2.2.2). Il est indispensable d'identifier chaque flacon de recueil, et chaque tube à centrifuger avec le nom du patient ou les deux noms des membres du couple s'ils sont différents.

L'analyse des paramètres spermatiques le jour $\mathrm{du}$ recueil est indispensable avec, au minimum, l'évaluation de la numération et de la mobilité des spermatozoïdes, et une numération des leucocytes. Si nécessaire, un deuxième recueil pourra être demandé. La préparation peut se faire selon différentes techniques sélectionnées en fonction des paramètres spermatiques : lavage - migration ascendante, gradients de densité. Il est nécessaire de faire l'analyse des paramètres après préparation afin d'évaluer le nombre de spermatozoïdes mobiles inséminés.

Si le sperme doit être transporté, cela doit se faire dans un système permettant le maintien du prélèvement entre $20^{\circ}$ et $35^{\circ}$ en évitant les variations thermiques. L'IAC est généralement réalisée avec au moins 500000 spermatozoïdes mobiles concentrés dans un volume de 0,2 à $0,3 \mathrm{ml}$ de milieu.

\subsection{LA FÉCONDATION IN VITRO INTRA- CONJUGALE (FIV-C)}

\subsection{1. - ASPECTS CLINIQUES}

\section{a) Indications}

La fivete (fécondation in vitro et transfert embryonnaire), avec ou sans micromanipulation, demeure une technique lourde et coûteuse dont les résultats restent limités. Ses indications peuvent être absolues (absence ou obstruction tubaire bilatérale, azoospermie...) ou relatives (altération tubaire, endométriose, insuffisance spermatique, idiopathique etc.). Dans les indications relatives, la FIV doit être proposée avec discernement, ni trop précocement avant d'avoir mis en œuvre d'autres pos- sibilités thérapeutiques, ni trop tardivement lorsque l'âge de la femme grèvera considérablement les chances de succès. Exceptionnellement, la FIV peut être proposée dans le but de réaliser un diagnostic biologique à partir de cellules prélevées sur l'embryon in vitro (article L. 162-17 CSP).

Enfin, un cycle de ponction ne doit pas être débuté tant que le couple dispose d'embryons congelés provenant d'une ponction antérieure.

\section{b) Stimulation}

La stimulation ovarienne vise à obtenir un nombre raisonnable d'ovocytes matures, le nombre habituel varie de 4 à 10 . L'obtention d'une moyenne différente doit faire réévaluer les protocoles utilisés. Elle sera modulée en fonction du statut ovarien de la femme. Il faudra également tenir compte des souhaits du couple (refus de congélation embryonnaire par exemple qui doit conduire à une stimulation si possible paucifolliculaire). La surveillance de cette stimulation repose sur un suivi échographique et hormonal.

La surveillance échographique se fait de préférence par voie vaginale à l'aide de sondes à haute définition. La mesure des diamètres folliculaires est répétée en fonction de la réponse de chaque patiente : 3 ou 4 échographies sont en général suffisantes. L'étude de l'épaisseur et des caractéristiques de l'endomètre peut $\mathrm{y}$ être associée.

La surveillance hormonale s'effectue par le dosage de l'oestradiol. Trois ou quatre dosages sont généralement réalisés avant la décision du déclenchement. Le dosage de la progestérone en fin de stimulation peut être utilisé pour affiner les critères de la maturation folliculaire. Le dosage de la LH n'est utile qu'en cas de stimulation n'associant pas un analogue de LHRH. Les autres dosages n'ont pas d'intérêt en pratique clinique habituelle.

Les modalités de la stimulation et de sa surveillance, de son arrêt éventuel, la décision de déclenchement de l'ovulation restent sous la responsabilité d'un clinicien agréé. En cas de réponse ovarienne excessive, le déclenchement de l'ovulation doit être annulé et la femme doit continuer à être suivie. 


\section{c) Ponction folliculaire}

La ponction folliculaire est réalisée, sous la responsabilité d'un praticien agréé, 33 à 36 heures après le déclenchement de l'ovulation, généralement sous échographie, par voie vaginale. Une anesthésie loco-régionale ou générale est pratiquée, en tenant compte du souhait de la patiente. Un anesthésiste doit être immédiatement disponible, même en cas d'anesthésie loco-régionale, permettant si nécessaire, la réalisation d'une anesthésie générale. Du fait de cette éventualité, une consultation pré anesthésique est recommandée. Les milieux de rinçage utilisés pour la ponction folliculaire sont choisis parmi les produits répondant aux normes en vigueur. Le maintien à $37^{\circ} \mathrm{C}$ du prélèvement doit être assuré dès la ponction jusqu'à son traitement au laboratoire.

\subsection{2. - ASPECTS BIOLOGIQUES}

Le traitement de gamètes et d'embryons humains dans le laboratoire nécessite le respect de règles de sécurité explicités au chapitre 1.1.2. Pour des raisons sanitaires et afin d'éviter tout risque d'erreur, les produits venant de couples différents ne doivent pas être traités par la même personne simultanément. Lidentification des gamètes et des embryons est d'une importance capitale et implique la vérification de l'identité des 2 membres du couple. Par la suite, chaque flacon de recueil, tube ou boite de culture utilisé doit porter le nom d'usage du couple (ou les 2 noms s'ils sont différents).

\section{a) Milieux de culture et matériel}

Les conditions de culture optimales imposent la maîtrise de plusieurs paramètres notamment : l'asepsie, la température et le $\mathrm{pH}$.

Les manipulations doivent être réalisées sous enceinte stérile à flux adapté, avec du matériel stérilisé sans substance connue comme toxique (comme l'oxyde d'éthylène) et des milieux de culture adaptés. Le personnel doit porter tenues à usage unique ou stérilisées quotidiennement, ainsi qu'un masque et des gants (Cf. 1.1.2.).

La température est maintenue à $37^{\circ} \mathrm{C}$ pendant toute la durée des manipulations et des transports éventuels des ovocytes et embryons : préchauffage du matériel plastique et des milieux de culture, utilisation d'un plateau chauffant dans la hotte, d'une platine chauffante sur le stéréomicroscope, d'une platine chauffante ou d'une enceinte thermostatée pour le microscope inversé, d'un bain-marie sec et d'un incubateur.

Le $\mathrm{pH}$ doit être maintenu autour de 7,2 grâce à une atmosphère gazeuse enrichie en $\mathrm{CO} 2$ pendant toute la durée de la culture.

Plusieurs milieux de culture actuellement disponibles dans le commerce ont montré une efficacité comparable. Néanmoins, ne peuvent être utilisés que ceux répondant aux exigences réglementaires en vigueur. En cas de supplémentation, ne peuvent être utilisés que des produits biologiques provenant du couple ou des substances commercialisées satisfaisant aux réglementations. L'utilisation de produits biologiques provenant d'autres patients est interdite.

Les milieux préparés au laboratoire doivent inclure des composés conformes aux normes, hautement purifiés et, lorsque cela est possible, spécifiques pour culture cellulaire. Un cahier de préparation doit être tenu comportant la composition du milieu, l'origine des composants et leur numéro de lot. De plus, un numéro de lot doit être attribué à chaque préparation de milieu. Toute nouvelle modalité de culture ou de conservation des embryons ne peut être pratiquée que dans le cadre d'une étude sur l'embryon autorisé par le Ministre chargé de la Santé après avis de la CNMBRDP (articles L. 152-8 et R. 152-8-3 du CSP). Dans tous les cas, les numéros de lot des milieux utilisés doivent figurer sur un document permettant d'assurer la traçabilité des milieux. En cas de culture sous huile, celle-ci doit être spécifique pour la culture cellulaire.

Le matériel plastique utilisé pour toutes les manipulations doit être non cytotoxique et, lorsque cela est possible, spécialement conçu pour culture cellulaire.

\section{b) Technique de FIV}

Le recueil du sperme doit se faire au laboratoire d'AMP autorisé notamment pour s'assurer de l'origine des gamètes. Lorsque des problèmes de recueil sont connus le sperme pourra être congelé en dehors de toute tentative de FIV de manière à être disponible en cas d'échec 
de recueil le jour de la tentative (Cf. autoconservation, 2.6.).

La préparation du sperme peut se faire selon différentes techniques sélectionnées en fonction de sa qualité : lavage simple, lavage et migration ascendante, gradient de densité ou toute autre méthode éprouvée.

L'insémination des ovocytes non atrétiques est réalisée dans les heures qui suivent le recueil ovocytaire avec un nombre de spermatozoïdes mobiles compris, sauf exception, entre 50000 et $200000 / \mathrm{ml}$. En cas d'asthénospermie, les délais entre le recueil de sperme, sa préparation et l'insémination doivent être les plus courts possibles.

Lorsque le couple refuse la congélation embryonnaire le nombre d'ovocytes inséminés doit être limité à 3 , le couple en ayant été averti au moment du consentement.

Les ovocytes sont examinés 16 à 20 heures après l'insémination de manière à évaluer la fécondation. La culture embryonnaire est ensuite poursuivie et le transfert envisagé pour ceux présentant au plus 2 pronuclei. La culture est poursuivie le plus souvent jusqu'à J2 ou J3.

Elle peut être parfois prolongée jusqu'à J5 - J7, soit par coculture, soit en utilisant des milieux synthétiques séquentiels, pour permettre le développement de l'embryon jusqu'au stade de blastocyste et ainsi un transfert plus tardif.

La coculture consiste à cultiver des embryons sur un tapis cellulaire. Seules les cellules autologues (cumulus, granulosa, endomètre de la patiente) n'induisent pas de risque infectieux exogène. L'usage des cellules d'une autre patiente est strictement interdit. Certains types de cellules (cellules Véro de rein de singe), ont pu être utilisées, mais n'ont fait actuellement l'objet d'aucune procédure de validation au regard de la sécurité sanitaire, en l'état de la législation actuelle.

Les techniques d'assistance médicale à la procréation innovantes non consacrées par l'usage, aboutissant à la formation d'embryons doivent être réalisées dans le cadre d'un projet de recherche clinique et après avis de la CNMBRDP.
Il convient de rappeler que lorsque ces techniques non éprouvées concernent l'embryon lui-même, elles entrent dans la procédure obligatoire d'autorisation d'études sur l'embryon humain (article L. 152-8 et R. 152-8-1 à R. 1528-12 du code de la santé publique).

\subsection{3 - TRANSFERT EMBRYONNAIRE}

Au moment du transfert embryonnaire, le clinicien et le biologiste doivent s'assurer personnellement de l'identité de la patiente. Les modalités du transfert s'appuient sur les données cliniques de la patiente et les résultats d'un test de transfert préalable dont les résultats sont consignés dans le dossier d'AMP. Enfin dans ces cas exceptionnels, il doit être possible de recourir à l'anesthésie générale.

\section{a) Choix des embryons à transférer}

Ce choix se fait en fonction des critères suivants :

- Normalité de la fécondation (présence de 2 pronuclei au maximum à J1),

- Cinétique embryonnaire, le meilleur développement étant 4 cellules à J2 et 8 cellules à J3,

- Morphologie embryonnaire évaluée sur l'homogénéité des blastomères et la proportion du volume des exsudats qui ne doit pas excéder $50 \%$.

Le choix des embryons à transférer est sous la responsabilité du biologiste et doit être explicité au couple. Selon leur aspect morphologique, le biologiste peut décider de ne pas transférer ni congeler les embryons obtenus.

\section{b) Nombre d'embryons à transférer}

Le nombre d'embryons à transférer doit être discuté conjointement entre le couple, le clinicien et le biologiste. Ce nombre dépend de l'aspect des embryons, de l'âge de la patiente et de ses antécédents. Il doit être autant que possible limité à 2 . Au delà, les raisons doivent être justifiées dans le dossier de la patiente.

c) Les couples doivent être informés des chances de succès ainsi que du risque de grossesse multiple et de tout incident sérieux survenu au cours des phases clinique et biologique de leur tentative et pouvant compromettre leurs chances de succès. Ces éléments doivent 
être mentionnés sur le dossier de la patiente et sur le registre des tentatives.

\subsection{FIV AVEC MICRO-INJECTION (ICSI)}

\subsubsection{Aspects cliniques}

L'ICSI doit être réservée à des cas où la fécondation in vitro par les techniques conventionnelles est impossible ou a de faibles chances de réussite. Il s'agit surtout des infécondités masculines sévères. Elle peut être envisagée en première intention dans les cas suivants :

- azoospermies impliquant un prélèvement épididymaire ou testiculaire des spermatozoïdes,

- oligo et/ou asthéno et/ou térato-zoospermie majeure,

akinétospermie,

certaines auto-immunisations anti-spermatozoïdes

En dehors de ces cas, l'indication doit se discuter en fonction du taux de fécondation obtenu lors des tentatives précédentes réalisées par FIV conventionnelle. Le choix de la technique et ses raisons seront alors clairement explicitées dans le dossier médical.

Un dossier-guide spécifique de l'ICSI, précisant les risques, notamment de transmission de la stérilité masculine, sera remis au couple. L'ICSI doit faire l'objet d'un consentement éclairé, signé, spécifique.

Enfin, les centres doivent collecter les données sur l'état de santé des enfants à la naissance. L'étude du devenir à long terme des enfants doit être encouragée.

\subsection{2. - Aspects biologiques}

Par rapport à la FIV classique, trois étapes sont spécifiques de l'ICSI, la préparation des spermatozoïdes, celle des ovocytes et la fécondation assistée elle-même.

\section{a) Préparation des spermatozoüdes}

La préparation des spermatozoïdes éjaculés ou recueillis par prélèvement épididymaire dépend de leur concentration. Il peut être demandé plusieurs éjaculats. Au dessus de 0,5 millions de spermatozoïdes mobiles par ml, les techniques classiques peuvent être utilisées (Cf. chapitre sur les techniques de FIV
2.2.2.). Au dessous de cette limite, un simple lavage-centrifugation de la totalité de l'éjaculat est réalisé.

En cas de ponction testiculaire, la biopsie est disséquée, de manière à recueillir les spermatozoïdes .

Lorsque les spermatozoïdes sont prélevés chirurgicalement, ceux qui ne sont pas utilisés doivent pouvoir être congelés, de manière à éviter une nouvelle ponction en cas d'échec de la tentative, après accord préalable du patient.

Dans l'état des connaissances actuelles, l'injection de cellules germinales immatures (spermatides) ou de spermatozoïdes dont la vitalité n'est pas établie ne peut être envisagée que dans un protocole de recherche clinique et après avis de la CNMBRDP.

\section{b) Préparation des ovocytes}

Les ovocytes prélevés en vue d'ICSI doivent être décoronisés de manière à visualiser l'introduction de la micropipette et l'injection du spermatozoïde. La décoronisation se fait par la hyaluronidase. Elle doit être rapide et suivie de plusieurs rinçages pour éviter une action délétère de l'enzyme. Elle permet de connaître l'état et la maturité des ovocytes qui doivent être notés sur le dossier biologique.

\section{c) Injection du spermatozoïde}

Le matériel nécessaire se compose d'un microscope inversé équipé de micro-injecteurs. Il doit être placé dans des conditions garantissant l'asepsie des manipulations (de préférence hotte à flux laminaire, salle stérile). Il est préférable d'utiliser les pipettes de maintien et d'injection commercialisées. Toutefois, lorsqu'elles sont fabriquées au laboratoire, elles doivent être nettoyées et stérilisées avant usage.

Linjection se déroule dans une boite de Pétri, sous huile (spéciale pour culture cellulaire). Le sperme est le plus souvent placé dans une goutte de polyvinylpyrolidone (PVP), de manière à ralentir le mouvement spermatique et faciliter l'injection, mais l'utilisation du PVP n'est pas indispensable. Le spermatozoïde est immobilisé par effet de cisaillement du flagelle. L'injection elle-même se fait au centre de l'ovocyte, ce dernier ayant été orienté de sorte 
que le globule polaire soit à $6 \mathrm{H}$ ou $12 \mathrm{H}$, donc à distance du fuseau méiotique. Elle doit être rapide, non traumatisante pour l'ovocyte. Les ovocytes sont ensuite remis en culture. Il est actuellement admis que $10 \%$ environ des ovocytes sont endommagés. Au delà de $15 \%$, il est nécessaire de revoir la technique.

Le reste de la technique se déroule de la même manière que pour la FIV. Cependant la coculture ne doit pas être associée à l'ICSI.

Le traitement d'une partie des ovocytes avec et sans micromanipulation doit rester exceptionnel et documenté dans le dossier. S'il a été décidé d'entreprendre une association "FIV classique -ICSI", et que des embryons sont issus des 2 techniques, seront transférés en priorité ceux provenant de FIV classique. Ils ne doivent pas être transférés simultanément (sauf justification dans le dossier), en raison notamment de la nécessité d'avoir un suivi correctement documenté des enfants qui en sont issus .

\section{4. - CONGÉLATION DES EMBRYONS}

Une bonne pratique de la FIV implique la possibilité de congeler des embryons en vue de les transférer ultérieurement. Cette possibilité est soumise au consentement écrit du couple.

\subsection{1. - INDICATIONS}

L'indication principale concerne les cas où le nombre d'embryons obtenus est supérieur au nombre d'embryons transférés. La congélation peut aussi être proposée s'il est nécessaire de retarder le transfert pour raisons médicales (maladie, hyperstimulation, traitement de chimiothérapie ou de radiothérapie, etc.), ou pour remplir les conditions de sécurité sanitaire (notamment dans le cadre du don d'ovocyte). Elle est enfin réalisable dans le cas d'impossibilité d'effectuer le transfert (accident, contretemps, etc.). L'indication doit toujours figurer dans le dossier.

\subsection{2. - ASPECTS CLINIQUES}

Le transfert d'embryons décongelés peut être réalisé lors de cycles spontanés, stimulés ou substitués. Lors des cycles spontanés le repérage de l'ovulation se base sur la mise en évidence de la décharge de LH. L'ovulation peut également être déclenchée lorsque le follicule a atteint $20 \mathrm{~mm}$ de diamètre.
La pratique d'une stimulation ovarienne implique un monitorage échographique et hormonal. La réponse ovarienne doit rester paucifolliculaire.

Les cycles substitués font appel dans un premier temps aux œstrogènes naturels. La progestérone ne sera administrée qu'après avoir vérifié l'absence d'ovulation spontanée.

Le délai entre le moment estimé de l'ovulation et le transfert doit être le même que celui écoulé entre la ponction et la congélation.

\subsection{3. - ASPECTS BIOLOGIQUES}

La congélation peut intervenir du stade des pronuclei (J1) au stade blastocyste (J6). Les embryons présentant plus de $30 \%$ de fragmentation ou un retard de développement ne doivent pas être congelés.

Le cryoprotecteur actuellement le plus efficace pour les embryons de 2 à 3 jours est le propanediol associé au sucrose. Il est conseillé de ne mettre qu'un seul embryon par paillette. On ne doit jamais en mettre plus de 2 afin de maîtriser au mieux le nombre d'embryons transférés. Les paillettes doivent porter très lisiblement les indications permettant l'identification des embryons. Elles sont ensuite déposées dans un congélateur programmable avant d'être immergées dans l'azote liquide. L'induction de la cristallisation doit être parfaitement contrôlée.

La décongélation se fait par simple réchauffement à température ambiante. Le cryoprotecteur est éliminé par incubation des embryons dans des solutions de concentration décroissante en propanediol, jusqu'à élimination du cryoprotecteur. Les embryons sont transférés in utero dans les quelques heures qui suivent le réchauffement.

En moyenne, après réchauffement, plus des $2 / 3$ des embryons décongelés devraient présenter au moins $50 \%$ de blastomères intacts. $\mathrm{Si}$ tel n'est pas le cas, la procédure de congélation - décongélation doit être reconsidérée.

La technique de congélation - décongélation des blastocystes est différente : elle nécessite un autre cryoprotecteur, le glycérol.

Chaque cycle de congélation et de décongéla- 
tion doit être mentionné sur le registre de conservation des embryons (voir chapitre 1.1.3.)

\section{5. - RECUEIL DE SPERMATOZOIDES PAR PONCTION}

Ces ponctions ne peuvent être envisagées que sous la responsabilité d'un praticien agréé, compétent en andrologie (article R.152-9-3 du CSP). Le biologiste doit se trouver à proximité immédiate du bloc chirurgical, car c'est son appréciation du nombre et de la qualité des spermatozoïdes qui va guider le chirurgien dans la poursuite ou l'arrêt du prélèvement.

Les spermatozoïdes devront être transportés rapidement au laboratoire et traités sans délai.

Le prélèvement peut se faire à partir de l'épididyme, du déférent ou du testicule. Le prélèvement épididymaire peut être proposé en cas d'azoospermie obstructive, devant l'impossibilité ou l'échec de la chirurgie réparatrice. L'anéjaculation d'origine organique, en particulier chez les paraplégiques, représente une autre indication de cette technique après échec des thérapeutiques classiques (vibro-massage, électro-stimulation, stimulation pharmacologique). En cas d'anéjaculation d'origine psychogène, le prélèvement épididymaire n'est réalisé qu'après avis du psychiatre.

Le prélèvement testiculaire est proposé en cas d'azoospermie obstructive lorsque le prélèvement épididymaire est impossible (échec, obstacle en amont de l'épididyme), ou en cas d'azoospermie non obstructive.

Deux techniques de prélèvement épididymaire sont actuellement utilisées, sous anesthésie locale, loco-régionale ou générale : prélèvement après abord chirurgical de l'épididyme ou ponction transcutanée à l'aiguille fine.

En ce qui concerne le prélèvement testiculaire, si la ponction testiculaire à l'aiguille a pu être proposée, la réalisation d'une biopsie chirurgicale semble préférable. Elle permet en effet un meilleur contrôle de l'hémostase et le recueil d'un nombre plus élevé de spermatozoïdes. Ce dernier élément est de particulière importance en raison des progrès réalisés dans la congélation des spermatozoïdes testiculaires.

Un délai de 6 mois est nécessaire entre deux prélèvements.

\subsection{AUTOCONSERVATION DE GAMÈTES}

Ce chapitre concerne essentiellement l'autoconservation de sperme. En effet, la congélation d'ovocytes et les prélèvements de fragments d'ovaire en vue de conservation pour une éventuelle AMP ultérieure restent du domaine de la recherche et doivent donc rentrer dans le cadre d'un protocole de recherche et faire l'objet d'une demande à un CCPPRB.

\subsection{1.- Aspects cliniques}

L'autoconservation de sperme doit être systématiquement proposée aux patients avant toute thérapeutique ou intervention potentiellement stérilisante (chimiothérapie, radiothérapie, chirurgie....)

Elle peut aussi être préconisée avant ou au cours d'une tentative d'AMP.

Le patient doit être informé de la qualité du sperme conservé et du nombre de paillettes.

Le bilan du patient comprendra en particulier la recherche des maladies infectieuses transmissibles: VIH 1 et 2, Syphilis, Hépatites B et C.

La durée de la conservation est prévue pour un an renouvelable sur demande écrite.

Seul le déposant peut demander la restitution ou la destruction de ses paillettes. Dans ces cas, un document est alors signé par le patient et contresigné par le médecin biologiste.

L'utilisation ultérieure du sperme en vue d'un acte d'assistance médicale à la procréation ne pourra se faire que dans la mesure où seront remplies les conditions prévues par l'article L. 152-2 du code de la santé publique.

En cas de nécessité du déplacement des gamètes confiés à un laboratoire, celui-ci a l'obligation absolue d'en informer préalablement la personne qui lui avait confié la conservation de ses gamètes ainsi que du nouveau lieu de conservation (article R. 184-1-14 du CSP).

\subsection{2.- ASPECTS BIOLOGIQUES}

De nombreuses méthodes de congélation du sperme existent et ont prouvé leur efficacité. 
La congélation repose sur l'utilisation de cryoprotecteurs dont le plus fréquemment utilisé est le glycérol. Il est en général intégré dans des milieux plus complexes, auxquels il convient d'adjoindre des antibiotiques. La dilution dépend de la concentration spermatique avec des rapports allant de 1/1 à 1/3.

Le sperme est conditionné en doses d'insémination ou paillettes. Le remplissage des paillettes s'effectue de préférence à l'aide d'une machine automatique qui en soude les extrémités.

Lors de la conservation de sperme de patients à risque viral, des paillettes haute sécurité doivent être utilisées.

La descente en température est soit manuelle (dans les vapeurs d'azote liquide) soit, de préférence, automatisée à l'aide d'un congélateur programmable permettant une meilleure reproductibilité. Les paillettes sont ensuite conservées dans l'azote liquide à - $196^{\circ} \mathrm{C}$.

La décongélation s'effectue de préférence à $37^{\circ}$ C. Un test de tolérance à la congélation doit être effectué systématiquement pour chaque prélèvement afin d'en vérifier la qualité.

\section{3 - DISPOSITIONS SPECIFIQUES AU DON (IAD, FIV-D)}

\section{1. - CADRE LÉGISLATIF}

L'AMP avec don de gamètes s'entend de l'IAD et de la FIV avec tiers donneur - don de spermatozoïdes ou d'ovocytes -, (article L. 673-1 du CSP), le double don étant interdit (article L. 152-3 al.1 du CSP). L'AMP avec don de sperme doit être réalisée avec du sperme congelé (article L. 673-3 du CSP). En revanche le don d'ovocyte implique une fécondation in vitro dès leur recueil. Les embryons obtenus seront ensuite congelés afin de respecter les conditions sanitaires en vigueur (Cf. 1.2.4.).

Le recueil, le traitement, la conservation et la cession des gamètes en vue de don ne peuvent être pratiqués que dans un établissement ou organisme de santé public ou privé à but non lucratif autorisé à cette fin (articles L. 673-5 du CSP et 511-14 du code pénal).

Le don de gamètes ne peut être pratiqué que dans le respect des principes de gratuité (articles $16-5$ et 16-6 du Code civil, L. 665-13 du CSP et 511-9 du code pénal) et d'anonymat (articles 16-8 du Code civil, L. 665-14 du CSP et 511-9 du Code pénal), ce qui interdit tout accord préalable entre le couple receveur et un donneur désigné. En outre, il est interdit de subordonner le bénéfice de l'AMP, à la désignation d'un donneur par le couple receveur, même en faveur d'un couple tiers anonyme (articles L. 673-7 du CSP et 511-13 du code pénal). Cette dernière disposition n'interdit pas d'informer les couples receveurs potentiels sur les difficultés rencontrées pour disposer de donneurs de gamètes, et de les encourager à aider l'équipe dans cette recherche.

\subsection{DISPOSITIONS RELATIVES AU DONNEUR}

Le donneur (ou la donneuse) doit faire partie d'un couple ayant procréé (article L. 673-2 du CSP). Ses gamètes ne peuvent plus être utilisés lorsque l'équipe médicale a connaissance de la naissance de cinq enfants issus de ce donneur dans le cadre de l'AMP (article L. 6734 du CSP). L'utilisation de sperme frais ou de mélange de spermes d'origines différentes est interdite (articles L. $673-3$ et $511-12$ du Code pénal). Il en est de même, par assimilation, pour le don d'ovocytes : le transfert simultané d'embryons issus de donneuses différentes est interdit, pour permettre l'application de l'article L. 673-6 du CSP.

\subsection{1. - ENTRETIENS ET FORMALITÉS PRÉALABLES AU DON}

Le donneur (ou la donneuse) s'entretient avec les membres de l'équipe médicale (article $\mathrm{R}$. 673-5-5 du CSP) dans une pièce aménagée à cet effet (article R. 673-5-4 du CSP) lui garantissant confidentialité et anonymat. Il est informé des conditions légales du don (article R. 673-5-5-2 ${ }^{\circ}$ du CSP), notamment du principe d'anonymat et de ses corollaires (absence de responsabilité encourue, impossibilité d'établir un lien de filiation à l'égard de l'enfant à naître (article 311-19 du Code civil) et du principe de gratuité qui lui interdit de percevoir une rémunération (article L. 665-13 du CSP). Toutefois, le remboursement des frais engagés par le don peut lui être accordée. 
Le donneur est informé de la nature des examens à effectuer avant le don (article R. 673-5$5-3^{\circ}$ du CSP).

Il est informé également de la nécessité pour lui ainsi que pour son conjoint ou concubin de consentir par écrit au don de gamètes. Il devra également consentir à la conservation, sous forme rendue anonyme, d'informations à caractère personnel relatives à sa santé dans le dossier prévu à l'article R 673-5-8 du CSP. Le contenu de ce dossier, les conditions de sa conservation et de sa consultation lui sont également précisés.

Une information doit être donnée concernant les contraintes liées au recueil de gamètes (déplacements et contraintes pratiques).

Pour ce qui concerne le don d'ovocyte, la femme reçoit en outre une information détaillée sur les conditions de la stimulation ovarienne et $\mathrm{du}$ prélèvement ovocytaire, les risques et contraintes liées à cette technique. Afin d'éviter d'éventuelles pressions, si les ovocytes proviennent d'une patiente bénéficiant d'une FIV pour elle-même et non d'une ponction effectuée exclusivement en vue du don, la patiente doit avoir préalablement été informée précisément des conséquences, notamment de la perte de chances pour elle-même consécutive à ce don. Ce don ne peut être décidé au cours du cycle de ponction. Le consentement doit avoir été signé avant le cycle de FIV (en plus du consentement habituel pour FIV), être renouvelé avant chaque cycle d'AMP et être confirmé à chaque recueil.

Le praticien agréé vérifie que le donneur remplit les conditions légales (article R. 673-5-5-1 CSP). Il recueille par écrit les consentements. Le consentement peut être révoqué avant tout prélèvement (article L. 665-11 du CSP).

\subsection{2. - Critères d'acceptabilité des don- neurs}

Cette sélection s'opère en fonction des antécédents médicaux personnels et familiaux, des résultats des tests de dépistage sanitaires obligatoires, de l'analyse du sperme ou du bilan gynécologique. L'âge doit être pris en compte pour limiter les risques génétiques.

\section{a) Antécédents et enquête génétique}

Il est indispensable de procéder à un examen clinique des donneurs et des donneuses.

Une étude complète et documentée de l'arbre généalogique du donneur doit être réalisée pour éviter la transmission à l'enfant d'une anomalie génétique sévère. Toute suspicion d'anomalie doit inciter à rechercher des renseignements précis auprès du médecin traitant avec l'accord du patient. L'établissement du caryotype est préconisé. Si le donneur appartient à un groupe de population prédisposé à certaines pathologies, il est indispensable de rechercher l'hétérozygotie (hémoglobinopathies dans le bassin méditerranéen, en Afrique, etc.). Les donneurs à risque de transmission d'une anomalie génétique mineure peuvent être acceptés. Pour conclure le dossier avant d'exclure le donneur s'il existe un doute, il est recommandé de prendre l'avis d'un généticien.

\section{b) Analyse du sperme}

Le recueil doit être fait impérativement sur place dans les mêmes conditions que celles décrites au chapitre 2.1. Les paramètres du sperme sont analysés dans l'heure qui suit. L'utilisation du sperme congelé repose sur le test de tolérance à la congélation. Ainsi ne seront retenues que les paillettes dont le test aura montré qu'elles contiennent au moins $20 \%$ de spermatozoïdes de mobilité progressive et, au total, 8 millions de spermatozoïdes mobiles $/ \mathrm{ml}$.

\subsection{3. - CONSERVATION DES INFORMA- TIONS DU DONNEUR}

Le dossier du donneur, archivé dans des conditions garantissant la confidentialité, conformément à l'article R. 673-5-8 du CSP doit contenir, sous forme rendue anonyme, les antécédents personnels et familiaux, les résultats des tests de dépistage sanitaires obligatoires, le consentement écrit du couple donneur ainsi que le nombre d'enfants issus du don. S'il s'agit d'un don de sperme, le dossier comprend la date des dons, le nombre de paillettes conser- 
vées, la date des cessions, le nombre de paillettes cédées. S'il s'agit d'un don d'ovocytes, il comprend la date de la ponction et le nombre d'ovocytes donnés.

\subsection{DISPOSITIONS RELATIVES AU COUPLE RECEVEUR}

Outre le respect des conditions légales prévues à l'article L. 152-2 du CSP pour toute AMP (Cf. 1.2.1.), le couple receveur doit satisfaire, dans le cas de l'AMP avec don de gamètes, à des dispositions particulières concernant la procédure préalable et le consentement.

\subsubsection{PROCÉDURE PRÉALABLE}

L'équipe pluridisciplinaire, à laquelle doit s'adjoindre un médecin qualifié en psychiatrie ou un psychologue (article R. 673-5-6 du CSP), délivre une information spécifique décrivant la technique d'AMP avec don de gamètes envisagée ainsi que les principales dispositions légales relatives au don (gratuité, anonymat, filiation). Les conditions législatives et réglementaires relatives aux donneurs leur sont exposées (information, consentement, précautions d'ordre médical, critères de sélection et d'appariement). Les entretiens se déroulent dans une pièce aménagée à cet effet permettant le respect de la confidentialité (article $R$. 673-5-4 du CSP). Les conditions d'accueil des couples receveurs doivent rendre impossible la rencontre avec les donneurs. Dans le cadre du don d'ovocytes, les couples doivent être informés de la nécessité de recourir à la fécondation in vitro avec le sperme du conjoint de la receveuse, et par la congélation des embryons qui en sont issus, ainsi que du délai d'attente de 6 mois au minimum avant leur transfert, rendu nécessaire pour refaire les tests sanitaires chez la donneuse.

Le couple doit avoir dans tous les cas un ou plusieurs entretiens avec le psychiatre ou le psychologue qui lui expose les enjeux du don de gamètes et évoque avec lui les questions soulevées éventuellement pour l'information de l'enfant sur les circonstances de sa conception. L'opportunité d'une demande d'adoption doit être également envisagée. L'équipe pluridisciplinaire évalue les motivations du couple dans l'intérêt de l'enfant à naître. Un doute sérieux sur la santé psychique des demandeurs ou la validité de leurs motivations pourra conduire, après entretien avec le psychiatre ou le psychologue et concertation au sein de l'équipe, à leur proposer un délai de réflexion supplémentaire ou à ne pas accepter leur demande.

Un consentement est recueilli et confirmé dans les mêmes conditions que pour l'AMP avec les gamètes du couple, comportant notamment, dans le cadre du don d'ovocytes un consentement pour la congélation des embryons. De plus, le responsable de l'équipe doit exiger la production de l'acte de consentement effectué par déclaration conjointe devant un juge du tribunal de grande instance ou un notaire dans les conditions des articles 1157-2 et 1157-3 du nouveau code de procédure civile. Cet acte mentionne que les informations relatives à la filiation ont été données par le juge ou le notaire. Il doit être fait mention de la date et du lieu de la déclaration conjointe dans le dossier conservé par l'établissement. Le consentement interdit toute contestation relative à la filiation de l'enfant, à moins qu'il ne soit soutenu que cet enfant n'est pas issu de l'AMP ou que le consentement a été privé d'effet.

C'est pourquoi il convient d'être très attentif à la pérennité du consentement. En effet, outre l'éventuelle révocation par écrit, le consentement peut être privé d'effet à la suite de l'une des circonstances suivantes: décès de l'un des membres du couple, dépôt d'une requête en divorce ou en séparation de corps ou cessation de la communauté de vie. Dans ce cas, la filiation de l'enfant pourrait être remise en question.

\subsubsection{ATTRIBUTION ET CESSION DES GAMÈTES}

Un appariement entre le couple receveur et un donneur ou une donneuse est souhaitable, dans le but de ne pas introduire de caractères discordant entre le couple et l'enfant à naître. Il s'effectue en tenant compte des caractéristiques physiques et éventuellement des groupes sanguins du couple receveur.

Les gamètes provenant de don ne peuvent être délivrés qu'au praticien mettant en œuvre l'AMP (insémination artificielle ou FIV) ou au couple receveur (article R. 673-5-7 du CSP). 
Avant toute utilisation de gamètes de donneur et tout transfert d'embryons congelés issus de don d'ovocytes, le praticien devra prendre connaissance des éléments concernant les tests de sécurité sanitaire (article R. 673-5-13 du CSP).

Les gamètes et embryons issus d'ovocytes cédés doivent être accompagnés du document décrit à l'article R. 673-5-12 du CSP.

\subsubsection{ASPECTS CLINIQUES ET BIOLO- GIQUES}

\section{a) Don de sperme}

L'infertilité masculine demeure l'indication essentielle de l'insémination artificielle avec sperme de donneur (art. L. 152-2 du code de la santé publique alinéa 2). En pratique dans ce cas, sont regroupées les stérilités masculines vraies comme l'azoospermie mais aussi les hypofertilités masculines plus ou moins sévères pour lesquelles une assistance médicale à la procréation intra-conjugale ne peut aboutir ou n'a que de très faibles chances de réussite.

Elle peut aussi avoir pour objet d'éviter la transmission à l'enfant d'une maladie d'uneparticulière gravité (article L. 152-2 du code de la santé publique alinéa 2) ; cela concerne :

l'indication d'IAD pour cause génétique : elle permet d'éviter de transmettre à l'enfant une maladie génétique engageant son pronostic vital ou entraînant un handicap sévère (maladie dominante chez le père par exemple)

l'indication d'IAD pour séropositivité de l'homme (VIH) : elle reste la seule technique sans risque de contamination de l'enfant et de la partenaire.

La décision d'insémination artificielle avec sperme de donneur quelle qu'en soit l'indication ne peut être prise qu'après concertation de l'équipe pluridisciplinaire.
La technique d'insémination est décrite au chapitre 2-1-2.

La technique de congélation est décrite au chapitre sur l'autoconservation du sperme (Cf. 2.6.2.). Dans le cas du don, les paillettes sont préalablement codées de façon anonyme.

\section{b) Don d'ovocytes}

Les indications de cette procédure concernent des femmes en âge de procréer principalement, chez lesquelles l'ovulation est impossible : ménopause précoce, dysgénésie gonadique, castration chirurgicale, séquelles de chimiothérapie, de radiothérapie, etc.. L'absence ou l'insuffisance marquée de réponse ovarienne à la stimulation lors de plusieurs tentatives de FIV peut également constituer une indication du don d'ovocytes. Enfin le don d'ovocytes peut être proposé en cas d'anomalie génétique d'une particulière gravité transmissible.

En ce qui concerne la donneuse, il est particulièrement important de suivre des règles de grande prudence dans la stimulation ovarienne, pour ne pas l'exposer à des risques excessifs d'autant que cette dernière ne tire aucun bénéfice pour elle-même de la fécondation in vitro. Il faut de plus la mettre en garde sur les risques de grossesse spontanée pour ellemême. Les ovocytes d'une même donneuse pourront être utilisés pour plusieurs couples receveurs.

En ce qui concerne la receveuse, il est conseillé de ne pas transférer plus de 2 embryons, car, dans cette indication, les taux d'implantation sont élevés, et le risque de grossesse multiple important.

Il est aussi nécessaire d'insister sur l'importance de mettre en place des procédures permettant de garantir l'anonymat entre donneuses et receveuses. 